\title{
A Randomized Controlled Trial of Pretransplant Antiviral Therapy to Prevent Recurrence of Hepatitis C After Liver Transplantation*
}

\author{
Gregory T. Everson, ${ }^{1}$ Norah A. Terrault, ${ }^{2}$ Anna S. Lok, ${ }^{3}$ Del R. Rodrigo, ${ }^{4}$ Robert S. Brown, Jr., ${ }^{5}$ Sammy Saab, ${ }^{6}$ \\ Mitchell L. Shiffman, ${ }^{7}$ Abdullah M.S. Al-Osaimi, ${ }^{8}$ Laura M. Kulik, ${ }^{9}$ Brenda W. Gillespie, ${ }^{10}$ \\ and James E. Everhart ${ }^{11}$; and the Adult-to-Adult Living Donor Liver Transplantation Cohort Study
}

Hepatitis $\mathrm{C}$ virus (HCV) infection recurs in liver recipients who are viremic at transplantation. We conducted a randomized, controlled trial to test the efficacy and safety of pretransplant pegylated interferon alpha-2b plus ribavirin (Peg-IFN- $\alpha 2 b / R B V)$ for prevention of post-transplant $\mathrm{HCV}$ recurrence. Enrollees had HCV and were listed for liver transplantation, with either potential living donors or Model for End-Stage Liver Disease upgrade for hepatocellular carcinoma. Patients with HCV genotypes (G) $1 / 4 / 6$ (n = 44/2/ 1) were randomized $2: 1$ to treatment $(n=31)$ or untreated control $(n=16)$; HCV G2/3 $(n=32)$ were assigned to treatment. Overall, 59 were treated and 20 were not. Peg-IFN$\alpha 2 \mathrm{~b}$, starting at $0.75 \mu \mathrm{g} / \mathrm{kg} /$ week, and RBV, starting at $600 \mathrm{mg} / \mathrm{day}$, were escalated as tolerated. Patients assigned to treatment versus control had similar baseline characteristics. Combined virologic response (CVR) included pretransplant sustained virologic response and post-transplant virologic response (pTVR), defined as undetectable HCV RNA 12 weeks after end of treatment or transplant, respectively. In intent-to-treat analyses, 12 $(19 \%)$ assigned to treatment and $1(6 \%)$ assigned to control achieved CVR $(P=0.29)$; per-protocol values were $13(22 \%)$ and $0(0 \%)(P=0.03)$. Among treated G1/4/6 patients, 23 of 30 received transplant, of whom $22 \%$ had pTVR; among treated G2/3 patients 21 of 29 received transplant, of whom $29 \%$ had pTVR. pTVR was $0 \%, 18 \%$, and $50 \%$ in patients treated for $<8,8-16$, and $>16$ weeks, respectively $(P=0.01)$. Serious adverse events (SAEs) occurred with similar frequency in treated versus untreated patients (68\% versus $55 \% ; P=0.30$ ), but the number of SAEs per patient was higher in the treated group (2.7 versus $1.3 ; P=0.003)$. Conclusion: Pretransplant treatment with Peg-IFN- $\alpha 2 b$ / RBV prevents post-transplant recurrence of $\mathrm{HCV}$ in selected patients. Efficacy is higher with $>16$ weeks of treatment, but treatment is associated with increased risk of potentially serious complications. (НерATOLOGy 2013;57:1752-1762)

*This is publication 18 of the Adult-to-Adult Living Donor Liver Transplantation Cohort Study.

Abbreviations: A2ALL, Adult-to-Adult Living Donor Liver Transplantation Cohort Study; AE, adverse event; ANC, absolute neutrophil count; b-DNA, branchedchain DNA; CHC, chronic hepatitis C; CI, confidence interval; Cr, creatinine; CTP, Child-Turcotte-Pugh (class or score); CVR, combined virologic response; DDLT, deceased donor liver transplantation; DSMB, data and safety monitoring board; EPA, erythropoietin analog; GCRC, General Clinical Research Centers; G-CSF, granulocyte colony-stimulating factor; Hb, hemoglobin; HCC, hepatocellular carcinoma; HCV, hepatitis C virus; IFN, interferon; INR, international normalized ratio; ITT, intent to treat; LADR, low accelerating dose regimen; LDLT, living donor liver transplantation; LOD, limit of detection; LT, liver transplantation; MELD, Model for End-Stage Liver Disease; Peg-IFN- $\alpha 2 b$, pegylated IFN alpha-2b; PCR, polymerase chain reaction; PLT, platelet; PP, per protocol; $p T V R$, post-transplant virologic response; RBV, ribavirin; RCT, randomized, controlled trial; RNA, ribonucleic acid; SAE, serious adverse event; SC, subcutaneously; SD, standard deviation; SVR 12, pretransplant sustained virologic response; SVR, sustained virologic response; TMA, transcription-mediated amplification.

From the ${ }^{1}$ Section of Hepatology, University of Colorado Denver, Aurora, CO; ${ }^{2}$ Division of Gastroenterology, University of California, San Francisco, CA; ${ }^{3}$ Department of Internal Medicine, University of Michigan, Ann Arbor, MI; ${ }^{4}$ Department of Surgery, University of Michigan, Ann Arbor, MI; ${ }^{5}$ Department of Medicine and Surgery, Columbia University College of Physicians and Surgeons, New York, NY; ${ }^{6}$ Department of Medicine and Surgery, University of California, Los Angeles, CA; ${ }^{7}$ Department of Medicine, Virginia Commonwealth University, Richmond, VA; ${ }^{8}$ Department of Medicine, University of Virginia, Charlottesville, VA; ${ }^{9}$ Department of Medicine and Surgery, Northwestern University, Chicago, IL; ${ }^{10}$ Department of Biostatistics, University of Michigan, Ann Arbor, MI; and ${ }^{11}$ Division of Digestive Diseases and Nutrition, National Institute of Diabetes and Digestive and Kidney Diseases, National Institutes of Health, Bethesda, MD.

Received March 8, 2012; accepted June 11, 2012.

The results of this study were presented, in part, at the 60th Annual Meeting of the American Association for the Study of Liver Diseases, Boston, MA, October 30-November 1, 2009.

The study is registered in ClinicalTrials.gov (no. NCT00135798). 
$\mathrm{R}$ ecurrence of hepatitis $\mathrm{C}$ virus (HCV) infection is inevitable in viremic patients undergoing liver transplantation (LT). ${ }^{1,2}$ Aggressive recurrence of hepatitis $\mathrm{C}$ is associated with rapid progression to cirrhosis, graft failure, and death or need for LT. $^{3-5}$ Prevention of allograft reinfection by pretransplant antiviral therapy is one strategy for improving graft and patient outcomes in recipients transplanted for chronic hepatitis $\mathrm{C}$ (CHC).

Virologic response to pegylated interferon (Peg-IFN) and ribavirin (RBV) is reduced in cirrhosis. In the registration trials for Peg-IFN/RBV, the rates of sustained virologic response (SVR) were 5\%-15\% lower in patients with advanced fibrosis or cirrhosis. ${ }^{6-8}$ Likelihood of SVR further diminishes with increasing severity of liver disease as the result of poor tolerability, dose reductions, discontinuation of therapy, and intrinsically compromised response to Peg-IFN/RBV. ${ }^{\text {9- }}$

11 SVR was only demonstrated in $13 \%$ of patients with HCV genotype 1 and decompensated liver disease, two thirds of whom were treatment naïve. ${ }^{12}$

Despite the reduced rates of SVR among patients with advanced liver disease, on-treatment clearance of HCV ribonucleic acid (RNA) from blood can be achieved in $30 \%-40 \%$ of patients with HCV genotype 1 and $70 \%-90 \%$ of patients with HCV genotypes 2 or 3. In the setting of LT, rendering blood free of HCV RNA before transplantation could potentially limit the risk for recurrent HCV after LT. Five published reports have suggested that suppression of HCV RNA in patients with advanced disease is achievable, and that $20 \%-30 \%$ of treated patients may remain free of $\mathrm{HCV}$ infection after transplantation. ${ }^{12-16}$ None of these reports was a randomized trial, limiting conclusions regarding efficacy and, more important, safety.
Herein, we report the efficacy and safety of PegIFN/RBV to prevent the recurrence of $\mathrm{HCV}$ in a cohort of patients from the Adult-to-Adult Living Donor Liver Transplantation Cohort Study (A2ALL).

\section{Patients and Methods}

Study Patients. Patients were enrolled from October 2005 to January 2009 and were followed through December 2009. Two groups of adult patients with chronic HCV infection listed for LT were included: those who had a potential living donor and those with hepatocellular carcinoma (HCC) eligible for Model for End-Stage Liver Disease (MELD) waiting list upgrade. These two patient groups were considered the best candidates for pretransplant HCV treatment because they typically had less-severe liver decompensation and therefore would be predicted to better tolerate therapy. Another feature of these two groups is a relatively short, predictable duration of time on the waiting list that allowed for timing of treatment. Key inclusion criteria were stable clinical status, HCV RNA positive, MELD $\leq 20$, and anticipated time to transplantation of at least 12 weeks. The protocol allowed an investigator to petition for enrollment of a patient with a MELD score from 21 to 25 . A clinical oversight committee (G.E., A.L., and N.T.) was created to review the clinical information and determine eligibility for enrollment. Only 1 case (MELD 22) was approved and enrolled under this provision.

The main exclusion criteria were history of null response to a previous course of full doses of Peg-IFN and RBV for at least 12 weeks, symptomatic cardiovascular or psychiatric disease or serious systemic illness, active

\footnotetext{
The patients participating in this trial were enrolled in the National Institutes of Health (NIH)-sponsored Adult-to-Adult Living Donor Liver Transplantation Cohort Study. This study was supported by the National Institute of Diabetes and Digestive and Kidney Diseases (NIDDK) through cooperative agreements (NIDDK grant nos.: U01-DK62536, U01-DK62444, U01-DK62467, U01-DK62483, U01-DK62484, U01-DK62494, U01-DK62496, U01-DK62498, U01-DK62505, and U01-DK62531). The trial was also funded by research grants from Schering-Plough, through a cooperative research and development agreement with NIH-NIDDK, and through a clinical trial agreement between Ortho-Biotech and NIH-NIDDK. This study was supported by the NIDDKD through cooperative agreements (listed in parentheses). Additional support was provided by the Health Resources and Services Administration and the American Society of Transplant Surgeons.

Address reprint requests to: Gregory T. Everson, M.D., Section of Hepatology, University of Colorado Denver, 1635 North Aurora Court, B-154, Aurora, CO 80045. E-mail: greg.everson@UCDenver.edu; fax: 720-848-2246.

Copyright $\odot 2012$ by the American Association for the Study of Liver Diseases.

View this article online at wileyonlinelibrary.com.

DOI 10.1002/hep.25976

Potential conflict of interest: Dr. Everson advises, consults for, and received grants from Merck and Schering-Plough. Dr. Terrault consults for, and received grants from Roche and Genentech. Dr. Lok consults for and received grants from Schering-Plough, Merck, Roche, and Genentech. Dr. Brown consults for Merck. Dr. Saab owns stock in, consults for, advises, and is in the speakers' bureau of Bristol-Myers Squibb. He consults for, advises, and is on the speakers' bureau of Genentech and Merck. Dr. Al-Osaimi consults for, advises, is on the speakers' bureau of, and received grants from Merck and Vertex. He is on the speakers' bureau of and received grants from Idenix, Novartis, and Gilead. He also consults for Amgen and received grants from Genentech, Valeant, Intermune, Pharmasset, HemoLife Medical, Genzyme, Ortho Biotech, GalxoSMithKline, Sanofi-Aventis, Bayer Healthcare, Hyperion, Bristol-Myers Squibb, Vital, and Octapharma. Dr. Shiffman advises and received grants from Achillion, Boehringer-Ingelheim, Globeimmune, Inhibitex, Novartis, Vertex, and Zymogenetics. He consults for Biolex, Human Genome Sciences, Romark. He advises Pfizer and Janssen. He received grants from Idenix. He advises, is on the speakers' bureau of, and received grants from Schering-Plough, Gilead, and Bristol-Myers Squibb. He consults for, advises, and received grants from Conatus. He consults for, advises, is on the speakers bureau of, and received grants from Roche and Anandys. He is on the speakers' bureau of and advises Bayer.

Additional Supporting Information may be found in the online version of this article.
} 
substance abuse within the previous 6 months, severe cytopenias not responsive to either erythropoietin analog (EPA) or granulocyte colony-stimulating factor (G-CSF), and unstable clinical courses related to ongoing gastrointestinal bleeding, refractory encephalopathy, or HCC beyond Milan criteria. Exclusionary laboratory criteria were creatinine $(\mathrm{Cr}) \geq 2.2 \mathrm{mg} / \mathrm{dL}$, hemoglobin $(\mathrm{Hb})<10$ $\mathrm{g} / \mathrm{dL}$, absolute neutrophil count (ANC) $<750 / \mathrm{uL}$, and platelets (PLT) $<35,000 /$ uL before initiation of therapy. EPA and G-CSF were allowed before enrollment to achieve these laboratory entry criteria.

The protocol was approved by the institutional review boards at the participating institutions, and all subjects provided written informed consent.

Study Design. Eligible patients were enrolled at seven clinical centers; patients infected with genotypes 1/4/6 were randomized $2: 1$ to treatment or observation using a web-based interface stratified by clinical center, whereas those with HCV genotypes $2 / 3$ were assigned to treatment. The different approach by genotype reflected a known lower virologic response to Peg-IFN/RBV for genotypes $1 / 4 / 6$ than for genotypes $2 / 3$ as well as the need to include untreated controls to assess treatment risk. The targeted duration of therapy ( $\geq 12$ weeks) was anticipated to be necessary to achieve virologic response. The risk of deferring transplantation was also considered. The intent was to treat patients up to the time of transplantation or for a maximum of 48 weeks. Treatment assignments were not blinded at any stage.

Treatment, using a low accelerating dose regimen (LADR), was initiated with Peg-IFN- $\alpha-2 b(0.75 \mu \mathrm{g} /$ $\mathrm{kg} /$ week) and RBV (600 mg/day). Dose escalations were performed at weeks 1 (Peg-IFN $1.5 \mu \mathrm{g} / \mathrm{kg} /$ week and RBV $800 \mathrm{mg} /$ day), 2 (RBV $1.0 \mathrm{~g} /$ day), and 3 (RBV $1.2 \mathrm{~g} /$ day for patients who weighed more than $75 \mathrm{~kg}$ ) based upon patient tolerance and weekly blood counts. Dose escalation of Peg-IFN required ANC $>750 / \mu \mathrm{L}$ and PLT $>35,000 / \mu \mathrm{L}$; dose escalation of $\mathrm{RBV}$ required $\mathrm{Hb}>10 \mathrm{~g} / \mathrm{dL}$. Once a patient reached the target RBV dose of 1-1.2 g/day (approximately $10.6-13.2 \mathrm{mg} / \mathrm{kg} /$ day), no further increases in RBV dose were made. Subsequent doses of Peg-IFN and RBV were adjusted based upon adverse events (AEs), patient tolerability, and blood counts. If the highest tolerated dose of Peg-IFN was $<0.5 \mathrm{ug} / \mathrm{kg}$, Peg-IFN was permanently discontinued. EPA (PROCRIT 10,00040,000 U weekly subcutaneously; SC) and G-CSF (NEUPOGEN 150-300 $\mu \mathrm{g}$ SC up to three times/week) were allowed before and during antiviral treatment if $\mathrm{Hb}<12.5 \mathrm{mg} / \mathrm{dL}$ or ANC $<1,000 / \mathrm{uL}$, respectively.

Per protocol, antibiotic prophylaxis was required for patients randomized to treatment who had a history of spontaneous bacterial peritonitis or low protein ascites. After several serious infections, the protocol was amended to administer prophylactic antibiotics to all patients with current or past history of ascites.

Patients were observed every 2 weeks until week 12, then monthly until transplantation or completion of 48 weeks of treatment. Complete blood counts, international normalized ratio (INR) of prothrombin time, and chemistry profile were measured at each visit and more often when clinically indicated. For those completing 48 weeks of treatment or undergoing transplantation, follow-up was every 12 weeks for 24 weeks post-treatment or 48 weeks post-transplantation.

Efficacy Assessments. The primary endpoint was post-transplant virologic response (pTVR), defined as undetectable HCV RNA at week 12 after LT. Pretransplant SVR (SVR12) was defined as undetectable HCV RNA at week 12 after end of treatment. Combined virologic response (CVR) comprised both SVR12 and pTVR.

Patient management required that HCV RNA was quantified locally. Assays differed by clinical center; some assayed HCV RNA by polymerase chain reaction (PCR) assays with limit of detection (LOD) of $50 \mathrm{IU} /$ $\mathrm{mL}$, whereas others used branched-chain DNA (b-DNA) assays with LOD of $615 \mathrm{IU} / \mathrm{mL}$. Serum samples were also stored for subsequent HCV RNA measurement by a central laboratory. The latter samples were first analyzed by b-DNA assay, and all samples with undetectable results $(<615 \mathrm{IU} / \mathrm{mL})$ were then retested by transcription-mediated amplification (TMA) with LOD of 5 IU/ $\mathrm{mL}$. For data analysis, HCV RNA central laboratory results were supplemented with local results when samples were missing or insufficient for central testing.

Safety Assessments. Safety measures included physical examination, $\mathrm{AE}$ assessment, and laboratory monitoring. Cytopenias with $\mathrm{Hb}<8 \mathrm{~g} / \mathrm{dL}, \mathrm{ANC}<500 / \mathrm{uL}$, or PLT $<20,000 / \mathrm{uL}$ required treatment interruption or discontinuation. Serious adverse events (SAEs) included standard World Health Organization criteria and specific events related to cirrhosis or LT. SAEs were evaluated for relationship to antiviral treatment by the site principal investigators. Deaths were reviewed by an oversight committee (G.E., A.L., and N.T.) and the data and safety monitoring board (DSMB) for A2ALL to evaluate relationship, if any, to antiviral treatment. The DSMB reviewed safety data quarterly and met twice per year to review study progress.

Statistical Analyses. Efficacy was tested first using intent-to-treat (ITT) analyses and subsequently using per protocol (PP) analyses; safety analyses were conducted using PP analyses. Descriptive statistics were reported as mean and standard deviation (SD) or 
percentages, as appropriate. Treatment balance over clinical and laboratory characteristics was tested by Student's $t$ test for continuous variables and chi-square test for categorical variables. Variables predictive of pTVR were tested by logistic regression; covariates tested included baseline HCV RNA, HCV genotype, graft type, treatment duration, use of growth factors during treatment, and achievement of $80 \%$ target dose of Peg-IFN and RBV. Proportions of SAEs in treated and untreated patients were compared using two-sided Fisher's exact tests; SAE rates were compared using Poisson regression. The time to first HCV RNA negativity was estimated by Kaplan Meier method, with censoring at death, but not at transplant. The distributions of HCV RNA by treatment week were estimated using the reverse Kaplan Meier method to account for data below LOD. ${ }^{17}$

Sample size was calculated for patients with genotypes $1,4,5$, or 6 assuming a 2:1 randomization $(\alpha=$ $0.05)$ and two-sided testing to detect a difference in pTVR of $30 \%$ in treated versus $1 \%$ in control patients with $89 \%$ power ( $\mathrm{n}=84$ transplanted patients). This enrollment target was not achieved $(n=47$ with genotypes $1 / 4 / 6$ enrolled, 35 transplanted), and recruitment was terminated to allow complete follow-up of enrolled patients before the end of funding. This analysis combines genotypes $2 / 3$ with genotypes $1 / 4 / 6$. Although an interim analysis was planned after half of the expected genotype $1 / 4 / 5 / 6$ patients were transplanted $(n=40)$, this analysis was not carried out because of low enrollment. Statistical analyses were performed using SAS 9.2 software (SAS Institute Inc., Cary, NC), with tests performed at a significance level of 0.05 .

\section{Results}

A total of 145 patients with chronic HCV, including 92 with HCC, were potentially eligible for this study. Eighty-seven (60\% of all eligible HCV cases), including 49 with HCC ( $53 \%$ of the HCC cases), were enrolled; 8 were screen failures and 79 were assigned to treatment groups (Fig. 1). Reasons for failure to enroll the remaining 58 patients were intolerance to previous interferon (IFN), unwillingness to take IFN-based treatment, null response to previous therapy, "too sick," contraindications to IFN or RBV, or inability to comply with visit schedule or study protocol.

Patients with HCV genotypes 1/4/6 ( $\mathrm{n}=44 / 2 / 1)$ were randomized $2: 1$ to treatment $(\mathrm{n}=31)$ or untreated control $(\mathrm{n}=16)$. HCV genotypes $2 / 3(\mathrm{n}=$ 32) were all assigned to treatment. Overall, 59 were treated with Peg-IFN/RBV and 20 were not treated. Of the 47 patients with $\mathrm{HCV}$ genotype 1/4/6, 2

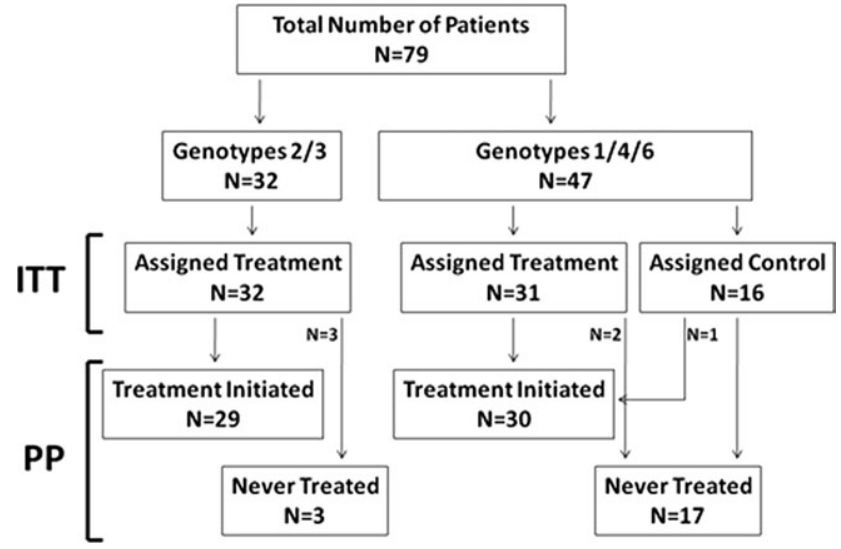

Fig. 1. Enrollment flow chart by HCV genotype, showing both ITT and treatment PP groups. All 32 patients infected with HCV genotypes 2 or 3 were assigned to treatment, but only 29 initiated treatment and 3 never received Peg-IFN or RBV. Forty-seven patients were infected with HCV genotypes 1, 4, or 6, of whom 31 were assigned to treatment and 16 were assigned to untreated control. Two of the patients assigned to treatment never received Peg-IFN or RBV, and 1 control was treated. The 8 screen failures are not reflected in this diagram.

patients assigned to treatment were never treated and 1 patient assigned to control was treated. Three of the thirty-two patients with HCV genotype $2 / 3$ did not receive treatment. Reasons for lack of treatment in the 5 patients assigned to treatment were early transplant, consent withdrawal, death, worsening renal function, and unknown. One control patient requested and received treatment off-protocol.

Baseline Characteristics. Demographic, clinical, and laboratory characteristics of the patients are shown in Table 1. The assigned treatment and control groups were well matched with respect to age, gender, ethnicity, race, weight, blood counts, baseline HCV RNA levels, and laboratory assessment of liver and renal function. In addition, mean ( \pm SD) MELD score (12.0 \pm 3.3 versus $12.0 \pm 3.8)$, and Child-TurcottePugh (CTP) mean $( \pm$ SD) score $(7.0 \pm 1.5$ versus $6.3 \pm 1.4)$ were similar. The treatment group had fewer patients with MELD upgrade for HCC (54\% versus $94 \% ; P=0.003)$. Sixty-two percent of treated patients and $56 \%$ of controls were IFN experienced. The duration of previous therapy and the type of virologic response to previous therapy (i.e., relapse, partial response, and null response) was not determined.

Virologic Response During Treatment with LADR. Cumulative distributions of HCV RNA results at baseline and during LADR treatment are given in Supporting Fig. 1 for HCV genotypes 1/4/6 (Supporting Fig. 1A) and HCV genotypes 2/3 (Supporting Fig. 1B). Patients with HCV genotypes $2 / 3$ had more rapid, greater suppression of HCV RNA during LADR treatment. 
Table 1. Baseline Characteristics of Patients by Assigned Treatment Group

\begin{tabular}{|c|c|c|c|}
\hline Characteristics & $\begin{array}{l}\text { Treatment }(n=63) \\
\text { Mean }(\mathrm{SD}) \text { or } \mathrm{N}(\%)\end{array}$ & $\begin{array}{c}\text { Control }(n=16) \\
\text { Mean }(S D) \text { or N (\%) }\end{array}$ & $P$ Value \\
\hline Age, years & $56(7.0)$ & $56(5.4)$ & 0.71 \\
\hline \multicolumn{4}{|l|}{ Gender (\%) } \\
\hline Male & $46(73)$ & $13(81)$ & 0.75 \\
\hline \multicolumn{4}{|l|}{ Ethnicity (\%) } \\
\hline Hispanic & $15(24)$ & $2(12)$ & 0.50 \\
\hline \multicolumn{4}{|l|}{ Race (\%) } \\
\hline White & $53(84)$ & $11(69)$ & \\
\hline African American & $3(5)$ & $2(12)$ & 0.28 \\
\hline Other & $7(11)$ & $3(19)$ & \\
\hline Weight (kg) & $84(15.3)$ & $88(13.5)$ & 0.31 \\
\hline BMI $\left(\mathrm{kg} / \mathrm{m}^{2}\right)$ & $28(4.4)$ & $29(4.9)$ & 0.45 \\
\hline \multicolumn{4}{|l|}{ HCV characteristics } \\
\hline \multicolumn{4}{|l|}{ Genotype (\%) } \\
\hline 1 & $30(47)$ & $14(88)$ & \\
\hline 2 & $16(24)$ & $0(0) *$ & $<0.001$ \\
\hline 3 & $16(25)$ & $0(0)^{*}$ & \\
\hline 4 or 6 & $1(4)$ & $2(12)$ & \\
\hline Viral load $\left(\log _{10} \mathrm{IU} / \mathrm{mL}\right)$ & $5.7(1.0)$ & $5.7(0.6)$ & 0.99 \\
\hline HCC upgrade (\%) & $34(54)$ & $15(94)$ & 0.003 \\
\hline \multicolumn{4}{|l|}{ Laboratory tests } \\
\hline Hemoglobin $(\mathrm{g} / \mathrm{dL})$ & $13.1(1.7)$ & $13.5(1.3)$ & 0.40 \\
\hline WBC $\left(\times 10^{3} / \mathrm{mm}^{3}\right)$ & $4.7(1.7)$ & $4.2(1.1)$ & 0.35 \\
\hline $\operatorname{ANC}\left(/ \mathrm{mm}^{3}\right)$ & $794(1,402)$ & $531(962)$ & 0.48 \\
\hline PLT $\left(\times 10^{3} / \mathrm{mm}^{3}\right)$ & $92(53)$ & $93(42)$ & 0.98 \\
\hline Albumin (g/dL) & $3.2(0.6)$ & $3.3(0.6)$ & 0.83 \\
\hline AST (IU/L) & $111(69)$ & $101(45)$ & 0.59 \\
\hline ALT (IU/L) & $84(59)$ & $79(40)$ & 0.74 \\
\hline Total bilirubin $(\mathrm{mg} / \mathrm{dL})$ & $2.1(1.4)$ & $2.2(1.6)$ & 0.73 \\
\hline Creatinine $(\mathrm{mg} / \mathrm{dL})$ & $0.9(0.3)$ & $0.9(0.2)$ & 0.81 \\
\hline INR & $1.3(0.2)$ & $1.3(0.2)$ & 1.00 \\
\hline MELD & $12.0(3.3)$ & $12.0(3.8)$ & 0.96 \\
\hline CTP Score & $7.0(1.5)$ & $6.3(1.4)$ & 0.11 \\
\hline Previous IFN treatment (\%) & $39(62)$ & $9(56)$ & 0.68 \\
\hline
\end{tabular}

Abbreviations: HCV, hepatitis C virus; HCC, hepatocellular carcinoma; ANC absolute neutrophil count; PLT, platelet; MELD, Model for End-stage Liver Disease; CTP, Child-Turcotte-Pugh (class or score); IFN, interferon; BMI, body mass index; WBC, white blood cell count; AST, aspartate aminotransferase; ALT, alanine aminotransferase; INR, international normalized ratio.

*By study design, all HCV genotype 2/3 patients were assigned to treatment.

Figure 2 shows time to first negative HCV RNA level during LADR treatment for patients achieving CVR. The probability of undetectable HCV RNA at week 16 was similar between patients with HCV genotypes 1/4/6 versus HCV genotypes 2/3 (log-rank test: $P=0.89$ ). Among patients who had CVR, all achieved undetectable HCV RNA by week 16 .

$\boldsymbol{C V R}$. For ITT analysis, $12(19 \%)$ of the 63 treatment-group patients and $1(6 \%)$ of the 16 controlgroup patients achieved CVR $(P=0.29)$ (Table 2). CVRs included 2 SVRs and 11 pTVRs. The single control CVR was the patient who requested and received treatment, achieved SVR, and did not undergo LT, despite being randomized to no treatment. For the PP analysis, 13 (22\%) of the 59 treated (95\% confidence interval $[\mathrm{CI}]: 0.12,0.35)$ and none of the untreated group achieved CVR $(P=0.03)$.
A higher proportion of patients with CVR had a $>2-\log _{10} \mathrm{IU} / \mathrm{mL}$ decrease in HCV RNA by treatment week $4(89 \%$ versus $68 \%)$ or week $8(100 \%$ versus $70 \%$ ) and undetectable HCV RNA by week 12 (100\% versus $32 \%)$, compared to patients not achieving CVR (Supporting Table 1).

LT. Fifty-seven patients, 44 treated and 13 controls, underwent LT: 16 living donor liver transplantations (LDLTs) and 41 deceased donor liver transplantations (DDLTs). Because of our selection criteria, more patients who underwent DDLT had HCC upgrade (90\% versus $6 \% ; P<0.0001)$. As a result, the DDLT group had lower laboratory MELD and CTP scores. Otherwise, the LDLT and DDLT groups had similar demographics, HCV genotype distribution, and mean baseline HCV RNA. Among those treated and transplanted, the proportions with pTVR were not significantly different: 5 of $16(31 \%)$ pTVR in LDLT versus 6 of $28(21 \%)$ in DDLT $(P=0.49)$.

Twenty-two patients were not transplanted and 7 of these had died, 1 each from liver failure, renal failure, multiorgan system failure, cardiac arrest, status epilepticus, sepsis, and unknown. Of the 15 who were alive at the end of follow-up, 2 had achieved SVR and were clinically stable, 8 were still listed and awaiting transplantation, 3 were delisted for progression of HCC, and 2 were delisted for severe deterioration in liver disease and clinical status.

Virologic Responses in Transplanted Patients. None of the 13 controls, but 26 of 44 (59\%) treated patients, achieved undetectable HCV RNA by the time of transplantation $(P<0.0001)$. Of the 44

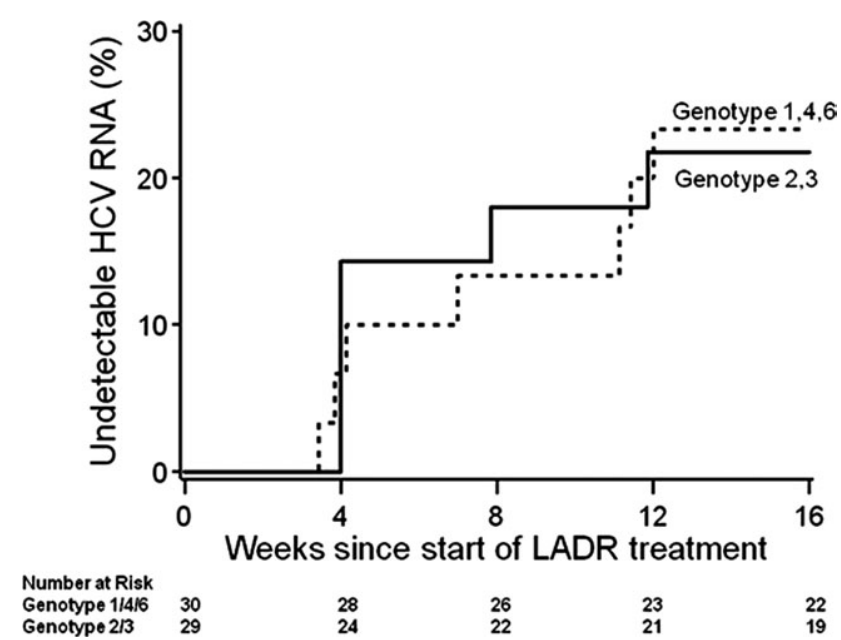

Fig. 2. Cumulative probability distribution, among treated patients, of time from study enrollment to first HCV RNA negativity for patients who achieved either SVR12 or pTVR, estimated by Kaplan-Meier. Dashed line shows the distribution for patients with HCV genotypes 1/ 4/6, and solid line for HCV genotypes 2/3. 
Table 2. Proportions and $95 \%$ Cls by Genotype of CVR* and pTVR

\begin{tabular}{lcccr}
\hline Genotype & Analysis & $\begin{array}{c}\text { CVR Among } \\
\text { All Patients }\end{array}$ & $\begin{array}{c}\text { pTVR Among } \\
\text { Transplanted Patients }\end{array}$ & $\begin{array}{c}\text { pTVR Among Transplanted Patients } \\
\text { HCV RNA Negative at Transplant }\end{array}$ \\
\hline $1 / 4 / 6$ & ITT & $6 / 31=0.19(0.07-0.37)$ & $5 / 24=0.21(0.07-0.42)$ & $5 / 12=0.42(0.15-0.72)$ \\
& PP & $7 / 30=0.23(0.09-0.42)$ & $5 / 23=0.22(0.07-0.44)$ & $5 / 12=0.42(0.15-0.72)$ \\
$2 / 3$ & ITT & $6 / 32=0.19(0.07-0.36)$ & $6 / 22=0.27(0.11-0.50)$ & $6 / 14=0.43(0.18-0.71)$ \\
& PP & $6 / 29=0.21(0.08-0.40)$ & $6 / 21=0.29(0.11-0.52)$ & $6 / 14=0.43(0.18-0.71)$ \\
& IIT & $12 / 63=0.19(0.10-0.31)$ & $11 / 46=0.24(0.13-0.39)$ & $11 / 26=0.42(0.23-0.63)$ \\
& PP & $13 / 59=0.22(0.12-0.35)$ & $11 / 44=0.25(0.13-0.40)$ & $11 / 26=0.42(0.23-0.63)$ \\
\hline
\end{tabular}

*Includes patients who achieved SVR (SVR12 and not transplanted) or pTVR.

Abbreviations: CVR, combined virologic response; SVR12, pretransplant sustained virologic response; pTVR, post transplant virologic response; ITT, intent to treat; PP, per protocol.

treated patients, 52\% with HCV genotypes $1 / 4 / 6$ and $67 \%$ with genotypes $2 / 3$ had undetectable HCV RNA at transplantation (Fig. 3).

The proportion with pTVR was comparable in ITT and PP analyses (Table 2). Eleven of the twenty-six $(42 \%)$ treated patients who had undetectable HCV RNA at transplantation achieved pTVR, 13 (50\%) relapsed, and $2(8 \%)$ died before week 12 post-transplant. None of the controls achieved pTVR $(P=0.03)$. pTVR did not differ between patients with HCV genotypes $1 / 4 / 6$ (5 of $23 ; 22 \%)$ versus patients with $\mathrm{HCV}$ genotypes $2 / 3$ (6 of $21 ; 29 \%)(P=0.60)$ (Fig. 3). All 11 patients with $\mathrm{pTVR}$ were retested at post-transplant week 24 and all remained HCV RNA undetectable.

The LOD of the test used to detect HCV RNA at transplant made a substantial difference in predicting pTVR. For the 26 patients with HCV RNA undetect-

\section{$\square$ Neg at LT $\quad$ Neg at 12 weeks Post-LT}

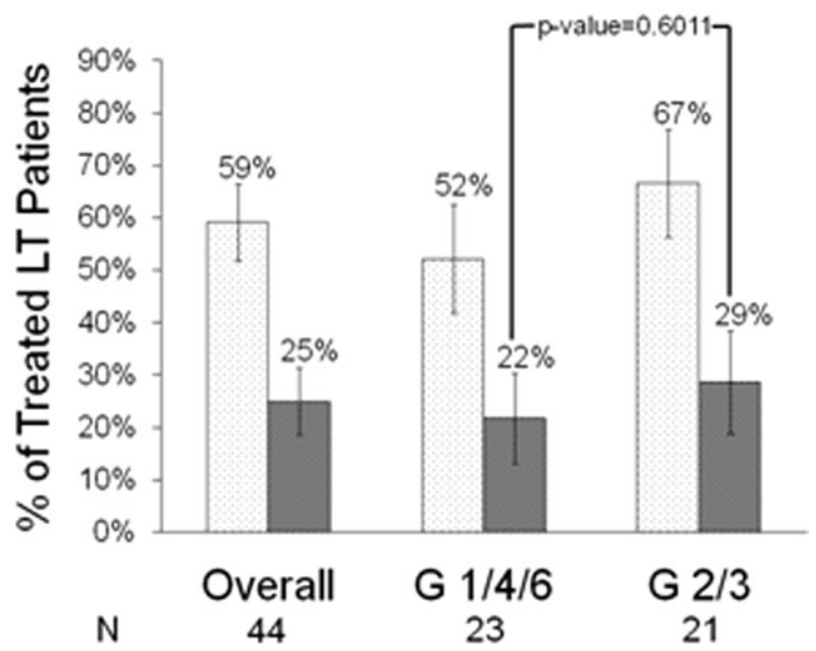

Fig. 3. Percent of treated patients with undetectable HCV RNA at transplant and at week 12 post-LT by genotype. Overall, 59\% of 44 treated patients were HCV RNA negative at time of transplant and $25 \%$ achieved pTVR (RNA negative at post-transplant week 12). Fifty-two percent of 23 genotype $(G) 1 / 4 / 6$ and $67 \%$ of $21 \mathrm{G} 2 / 3$ were RNA negative at transplant $(P=0.33)$; and $22 \%$ of $G 1 / 4 / 6$ and $29 \%$ of $G 2 / 3$ achieved pTVR $(P=0.60)$. PP analyses; whiskers are \pm 1 standard error. able at transplant, 8 were tested using assays with LOD of $5 \mathrm{IU} / \mathrm{mL}$, compared to 18 tested using assays with LOD $>5 \mathrm{IU} / \mathrm{mL}$, and the pTVR was $75 \%$ and $28 \%$, respectively, in these groups $(P=0.038)$.

Predictors of $p T V R$. Likelihood of pTVR increased with the duration of treatment. In patients who received $<8,8-16$, and $>16$ weeks of treatment, pTVR was $0 \%, 18 \%$, and $50 \%$, respectively $(P=$ 0.01) (Fig. 4). In univariate PP analyses, duration of pretransplant RBV (categorized; $P=0.01$ ) and PegIFN (categorized; $P=0.05$ ) were the only factors significantly associated with pTVR (Table 3). Although other factors were not significant, patients experiencing pTVR were more likely to be infected with HCV genotypes 2/3, had lower baseline HCV RNA, used growth factors during treatment, and achieved target doses of Peg-IFN and RBV.

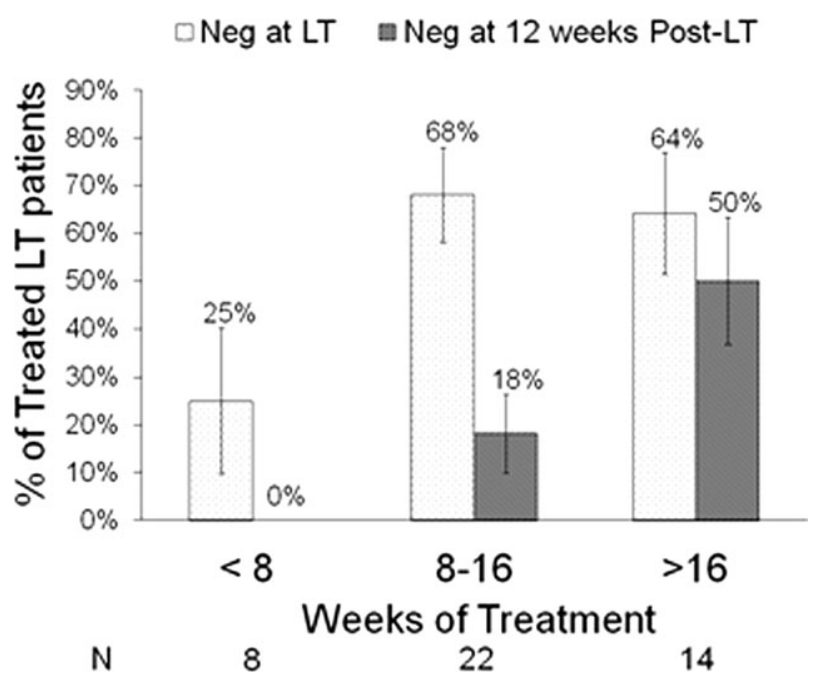

Fig. 4. Percent of treated patients with undetectable HCV RNA at transplant and at week 12 post-LT (pTVR) by treatment duration. Virologic response to pretransplant therapy was linked to treatment duration. Only $25 \%$ of the patients treated for less than 8 weeks had undetectable HCV RNA at transplant, and none achieved pTVR. In contrast, $64 \%$ of patients treated for more than 16 weeks had undetectable HCV RNA at transplant, and 50\% achieved pTVR. PP analyses; whiskers are \pm 1 standard error. 
Table 3. Predictors of pTVR Among Those Transplanted Who Were Assigned to Treatment $(\mathrm{ITT}, \mathbf{n}=46)$ or Who Received Treatment (PP, $n=44)$

\begin{tabular}{|c|c|c|c|c|c|c|}
\hline \multirow[b]{2}{*}{ Variables } & \multicolumn{3}{|c|}{ ITT analysis } & \multicolumn{3}{|c|}{ PP Analysis } \\
\hline & $\mathbf{O R}$ & $95 \% \mathrm{Cl}$ & $P$ Value & OR & $95 \% \mathrm{Cl}$ & $P$ Value \\
\hline Baseline HCV RNA $\log _{10} \mathrm{IU} / \mathrm{mL}$ & 0.54 & $0.26-1.12$ & 0.10 & 0.53 & $0.26-1.10$ & 0.09 \\
\hline Baseline HCV RNA $>5.5$ versus $\leq 5.5 \log _{10} \mathrm{IU} / \mathrm{mL}$ & 0.34 & $0.08-1.38$ & 0.13 & 0.29 & $0.07-1.19$ & 0.09 \\
\hline HCV genotype: $1 / 4 / 6$ versus $2 / 3$ & 0.70 & $0.18-2.74$ & 0.61 & 0.69 & $0.18-2.73$ & 0.60 \\
\hline LDLT versus DDLT & 1.81 & $0.46-7.26$ & 0.40 & 1.67 & $0.42-6.69$ & 0.47 \\
\hline \multicolumn{7}{|l|}{$\begin{array}{l}\text { Duration of pre-liver transplant treatment } \\
\text { Peg-IFN }\end{array}$} \\
\hline $\begin{array}{l}\text { Number of weeks (continuous) } \\
\text { Categorized }\end{array}$ & 1.09 & $0.99-1.19$ & 0.09 & 1.08 & $0.98-1.19$ & 0.14 \\
\hline$>16$ & 1 & & & 1 & & \\
\hline $8-16$ & 0.36 & $0.08-1.64$ & 0.03 & 0.36 & $0.08-1.64$ & 0.05 \\
\hline$<8$ & 0.11 & $0.01-1.11$ & & 0.13 & $0.01-1.35$ & \\
\hline \multicolumn{7}{|l|}{ RBV } \\
\hline $\begin{array}{l}\text { Number of weeks (continuous) } \\
\text { Categorized }\end{array}$ & 1.09 & $0.99-1.20$ & 0.07 & 1.09 & $0.97-1.20$ & 0.10 \\
\hline$>16$ & 1 & & & 1 & & \\
\hline $8-16$ & 0.24 & $0.06-1.08$ & 0.004 & 0.24 & $0.06-1.08$ & 0.01 \\
\hline$<8$ & 0.05 & $0.002-1.12$ & & 0.06 & $0.002-1.45$ & \\
\hline Use of growth factors during treatment & 4.58 & $0.52-40.38$ & 0.17 & 3.75 & $0.42-33.61$ & 0.24 \\
\hline Ability to achieve $80 \%$ of target dose of both Peg-IFN and RBV & 3.38 & $0.63-17.96$ & 0.15 & 2.93 & $0.54-15.75$ & 0.21 \\
\hline
\end{tabular}

Abbreviation: OR, odds ratio; pTVR, post transplant virologic response; ITT, intent to treat; PP, per protocol; HCV, hepatitis C virus; LDLT, living donor liver transplantation; DDLT, deceased donor liver transplantation; IFN, interferon; RBV, ribavirin.

SAEs and Death. SAEs before and up to 1 year after LT are listed in Table 4. There was no significant difference in the proportion of treated patients and controls that experienced SAEs $(68 \%$ versus $55 \% ; P=$ $0.30)$, but the number of SAEs per patient was greater in treated patients $(2.7$ versus $1.3 ; P=0.003)$. There was no association between MELD score and either the total number of SAEs or SAEs resulting from infection (data not shown).

Before LT, a greater proportion of treated patients had SAEs ( $46 \%$ versus $20 \% ; P=0.04)$. SAEs of cytopenia $(19 \%$ versus $0 \% ; P=0.06)$ and infection $(12 \%$ versus $0 \% ; P=0.18)$ were only observed in treated patients. Liver-related SAEs occurred with similar frequency (treated versus control, $14 \%$ versus $15 \% ; P=1.0$ ).

Infection was more common in treated patients. As mentioned above, 7 of 59 treated patients $(12 \%)$ and 0 of 20 controls $(0 \%)$ experienced an SAE of infection. Nineteen of fifty-nine treated patients $(20 \%)$ and 2 of 20 controls (10\%) experienced an $\mathrm{AE}$ of infection.

Rates of infection in treated patients may have been reduced by antibiotic prophylaxis. SAEs of infection occurred in $10 \%$ ( 5 of 48 ) of patients receiving prophylaxis, compared to $18 \%$ (2 of 11) not receiving prophylaxis. AEs of infection occurred in 29\% (14 of 48 ) of patients receiving prophylaxis, compared to $45 \%$ (5 of 11) not receiving prophylaxis.

SAEs also tended to be more common in treated patients early after transplantation. Within the first 30 days, a greater proportion of treated patients had
SAEs, compared to controls $(32 \%$ versus $15 \% ; P=$ 0.31 ), and infection was only observed in treated patients ( $9 \%$ versus $0 \% ; P=0.56)$.

Between 30 days and 1 year post-transplantation, we found that a similar proportion of treated patients and controls had experienced an SAE $(55 \%$ versus $46 \% ; P=0.59)$, but treated patients had more SAEs per patient $(2.1$ versus $1.2 ; P=0.06)$. Rejection within the first year occurred in 1 treated patient and in none of the control patients $(2 \%$ versus $0 \% ; P=$ 1.0).

Despite the greater risk for SAEs, pretransplant treatment was not associated with increased risk of death: $9(15 \%)$ treated patients and $2(10 \%)$ controls died (log-rank test: $P=0.81$ ). Five of the treated patients and 2 of the untreated patients died pre-LT. Overall, mortality rates were $7.0 \%$ (4 of 57) in transplant recipients, compared to $31.8 \%$ (7 of 22 ) in those without a transplant.

Dose and Duration of Peg-IFN and RBV. Patients achieving pTVR had higher exposure to both Peg-IFN and RBV. Cumulative doses per kilogram of body weight, duration of treatment, and percentages of patients achieving $80 \%$ of target doses for both PegIFN and RBV trended higher in the patients achieving pTVR (Supporting Table 2).

Growth Factors. Forty-four of the fifty-nine treated patients $(75 \%)$ received growth factors (9 received GCSF alone, 14 received EPA alone, and 21 received both G-CSF and EPA). Ten of the eleven patients 
Table 4. SAEs in Untreated and Treated Patients Pre-LT, in the First 30 Days Post-LT, and 30 Days to 1 Year Post-LT

\begin{tabular}{|c|c|c|c|}
\hline & Treated & Untreated & $P$ Value \\
\hline \multicolumn{4}{|l|}{ Overall } \\
\hline Number of patients with SAEs \% & $40 / 59(68)$ & $11 / 20(55)$ & 0.300 \\
\hline Total number of events & 109 & 14 & \\
\hline Number of SAEs/patient among those with SAEs & 2.7 & 1.3 & 0.003 \\
\hline Number of deaths* \% & $9 / 59(15)$ & $2 / 20(10)$ & $0.810 \dagger$ \\
\hline \multicolumn{4}{|l|}{ Pre-LT } \\
\hline Number of patients with SAEs $\%$ & $27 / 59(46)$ & $4 / 20(20)$ & 0.040 \\
\hline Total number of SAEs & 42 & 5 & \\
\hline Number of events/patient among those with SAEs & 1.6 & 1.3 & 0.520 \\
\hline \multirow[t]{3}{*}{ Cytopenia } & $11(19)$ & $0(0)$ & 0.060 \\
\hline & 6 & 0 & \\
\hline & 5 & 0 & \\
\hline \multirow[t]{3}{*}{ Infection } & $7(12)$ & $0(0)$ & 0.180 \\
\hline & 3 & 0 & \\
\hline & 4 & 0 & \\
\hline \multirow[t]{3}{*}{ Liver related } & $8(14)$ & $3(15)$ & 1.000 \\
\hline & 6 & 3 & \\
\hline & 2 & 0 & \\
\hline Other \% & $11(19)$ & $1(5)$ & 0.280 \\
\hline \multicolumn{4}{|l|}{ Post-LT, first 30 days } \\
\hline Number of patients with SAEs \% & $14 / 44(32)$ & $2 / 13(15)$ & 0.310 \\
\hline Total number of SAEs & 17 & 2 & \\
\hline Number of events/patient among those with SAEs & 1.2 & 1.0 & 0.460 \\
\hline Cytopenia \% & 0 & 0 & 1.000 \\
\hline Infection \% & $4(9)$ & 0 & 0.560 \\
\hline Liver related \% & $3(7)$ & $1(8)$ & 1.000 \\
\hline Rejection \% & $1(2)$ & 0 & 1.000 \\
\hline Surgical complication \% & $2(5)$ & 0 & 1.000 \\
\hline Other \% & $7(16)$ & $1(8)$ & 0.670 \\
\hline \multicolumn{4}{|l|}{ Post-LT, 30 days to 1 year } \\
\hline Number of patients with SAEs $\%$ & $24 / 44(55)$ & $6 / 13(46)$ & 0.590 \\
\hline Total number of SAEs & 50 & 7 & \\
\hline Number of events/patient among those with SAEs & 2.1 & 1.2 & 0.060 \\
\hline Cytopenia \% & 0 & $2(15)$ & 0.050 \\
\hline Infection \% & $9(20)$ & $3(23)$ & 1.000 \\
\hline Liver related \% & $2(5)$ & 0 & 1.000 \\
\hline Rejection \% & 0 & 0 & 1.000 \\
\hline Surgical complication \% & $8(18)$ & 0 & 0.180 \\
\hline Other \% & $13(30)$ & $2(15)$ & 0.480 \\
\hline
\end{tabular}

Abbreviation: SBP, spontaneous bacterial peritonitis; SAE, serious adverse events; LT, liver transplantation.

*Causes of death in these 11 patients were cardiac arrest $(n=2)$, sepsis $(n=2)$, heart failure $(n=2)$, liver failure $(n=1)$, renal failure $(n=1)$, cerebral edema $(n=1)$, and unknown $(n=2)$.

†Based on log-rank test.

achieving pTVR (91\%), compared to 24 of 33 patients without pTVR $(73 \%)(P=0.41)$, used growth factors during the course of pretransplant therapy (Supporting Table 2).

\section{Discussion}

LADR-A2ALL is the first randomized, controlled trial (RCT) of pretransplant treatment of $\mathrm{CHC}$ using Peg-IFN plus RBV to prevent recurrent HCV infection after transplantation. LADR-A2ALL sought to determine the efficacy of pretransplant treatment to prevent recurrent $\mathrm{HCV}$ and included an untreated control group to define the safety of Peg-IFN and RBV when used in this setting. Pretransplant treat- ment achieved post-transplant clearance of $\mathrm{HCV}$ pTVR in $25 \%$. However, treatment was associated with an increased frequency of SAEs, including infection.

In LADR-A2ALL, 59\% of patients were HCV RNA negative at the time of transplant. This rate of on-treatment viral clearance was higher than noted in previous reports using comparable doses of IFN, or Peg-IFN, with RBV. In the reports by Everson et al., ${ }^{12}$ Forns et al., ${ }^{14}$ and Carrion et al., ${ }^{16} 32 \%, 35 \%$, and $35 \%$ of patients were HCV RNA negative at the time of transplantation-approximately half the virologic response achieved in LADR-A2ALL. One contributing factor to the higher on-treatment virologic response may have been the relatively higher proportion of 
patients infected with HCV genotypes 2/3. The percentage of patients infected with HCV genotypes 2 or 3 in other studies ranged from $17 \%$ to $33 \% .{ }^{13-16,18}$ In LADR-A2ALL, $53 \%$ of treated patients were infected with HCV genotype 2 or 3. Another factor may have been the exclusion from the study of patients with previous null response to Peg-IFN and RBV.

The higher on-treatment virologic response in LADR-A2ALL could also have been the result of the use of a variety of HCV RNA assays, with LODs as high as $615 \mathrm{IU} / \mathrm{mL}$. A significant proportion of patients with undetectable HCV RNA by these insensitive assays were likely still viremic. This conclusion is supported by our TMA results, where pTVR was achieved in $75 \%$ of patients with undetectable HCV RNA by TMA, compared to only $28 \%$ of patients with undetectable HCV RNA, by the less-sensitive assays. This experience indicates that future treatment trials must use centralized sensitive assays to accurately define viral kinetics and virologic responses.

The percentage pTVR achieved in LADR-A2ALL $(25 \%)$ was similar to pTVR percentages achieved in the reports by Everson et al. (26\%), Forns et al. (23\%), and Carrion et al. (23\%). The relapse rate in LADR-A2ALL of $50 \%$ was higher than that observed by Everson et al. (20\%), Forns et al. (33\%), and Carrion et al. (33\%). ${ }^{12,14,16}$ The higher rate of relapse in LADR-A2ALL was likely related, at least in part, to the high proportion of patients who received a short duration of treatment. In our univariate analyses, the only factor predictive of pTVR was longer duration of pretransplant treatment. Shorter duration of treatment was associated with higher likelihood of relapse$100 \%$ of patients receiving less than 8 weeks of treatment relapsed.

Maintaining adequate doses and blood levels of RBV may be particularly important when using lower doses or dose reductions in Peg-IFN. ${ }^{18}$ In LADRA2ALL, patients experiencing pTVR were more likely to have achieved $80 \%$ of their targeted dose of RBV (91\% versus $70 \%$ ) and to have remained on RBV longer (17.3 versus 12.9 weeks). Patients achieving pTVR were more likely to have used EPA, a factor that could have allowed greater exposure to RBV and improved the likelihood of clearing HCV. ${ }^{19}$

Factors predicting virologic response in patients with advanced stages of $\mathrm{CHC}$ have been noted in other studies. Everson et al. found three factors predictive of SVR in 124 patients with advanced hepatitis C: infection with HCV genotype 2 or 3 (compared to genotypes 1/4/6), CTP class A cirrhosis (compared to CTP classes $\mathrm{B}$ and $\mathrm{C}$ ), and dose and duration of IFN (or Peg-IFN) and RBV treatment. ${ }^{12}$ Similar observations were made by Forns et al., Carrion et al., and Thomas et al. ${ }^{14-16}$ Carrion et al. also demonstrated that "early" virologic response $\left(>2 \quad \log _{10} \quad \mathrm{IU} / \mathrm{mL}\right.$ decrease by week 4) during treatment predicted likelihood for pTVR. ${ }^{16}$ In LADR-A2ALL, pTVR was only possible in the patients who were HCV RNA negative by week 12. The findings of Carrion et al. and LADR-A2ALL suggest that treatment could be discontinued in patients who either fail to achieve a $2-\log _{10}$ drop in HCV RNA by week 8 when using standard doses of Peg-IFN/RBV or who remain HCV RNA positive at week 12 when treated with LADR.

Pretransplant treatment with Peg-IFN plus RBV is only applicable to a select group of patients. We enrolled two types of patients into our study-potential recipients of LDLT who had an identifiable donor undergoing evaluation and potential recipients of DDLT who met criteria for MELD upgrade for HCC. These patients generally have less-severe liver disease, compared to patients awaiting DDLT for complications of liver failure, and are therefore able to better tolerate the AEs associated with Peg-IFN/RBV. Additionally, in these two patient groups, the interval between initiation of Peg-IFN/RBV treatment and transplantation is somewhat predictable. In the case of LDLT, the date of transplantation can be scheduled. In the case of DDLT for HCC after MELD upgrade, transplantation would typically occur within 6 months after the MELD upgrade. In some centers in the United States, LT may be performed at relatively low MELD scores, and the time interval between listing and transplantation can be estimated. Patients with HCV awaiting DDLT in these low-MELD centers might also be candidates for pretransplant treatment. However, practical logistical issues, such as underlying HCC and need for urgent transplantation or availability of donor liver, compromise the ability to extend treatment duration.

In LADR-A2ALL, treated patients experienced more SAEs and infection. In the case-control study of Carrion et al., ${ }^{16}$ treated patients had a significantly increased risk for bacterial infection $(P<0.001)$. The increased risk of infection indicates that prophylactic antibiotics during antiviral therapy may be warranted. Experience in both LADR-A2ALL and Barcelona suggested that antibiotic prophylaxis may have lowered the risk for bacterial infection during Peg-IFN/RBV treatment.

Nine treated patients (15\%) and 2 control patients $(10 \%)$ died. In the absence of a control group, the mortality rate in treated patients might be viewed as 
excessive and related to antiviral therapy. However, the similar mortality rate of controls suggests that our observed mortality rate might represent the underlying risk of mortality in these patients on the waiting list before and after hepatic transplantation. Support for this interpretation is provided by the case-control study of Carrion et al. ${ }^{16}$ Mortality rates were nearly identical to rates we reported in LADR-A2ALL-8 deaths in 51 treated patients $(16 \%)$ versus 7 deaths in 51 case controls (14\%).

Limitations of the study included failure to reach enrollment target, inability to complete planned minimum duration of treatment of 12 weeks for many subjects, partly resulting from the timing of DDLT availability, incomplete HCV RNA follow-up, inconsistency of LODs of HCV RNA assays, and noncompliance with assigned treatment in a few patients. Generalizability of results is primarily for the U.S. Caucasian population. Strengths of the study include randomization among HCV genotypes 1/4/6, allowing comparison of AEs between treated patients and controls.

In summary, we conducted an RCT of Peg-IFN and RBV in 79 patients with advanced hepatitis $C$ who were candidates for LT. Pretransplant treatment prevented post-transplant recurrence of $\mathrm{HCV}$ infection in $25 \%$ of transplanted cases-22\% in HCV genotypes $1 / 4 / 6$ and $29 \%$ in HCV genotypes $2 / 3$. The strongest predictor of virologic response was duration of treatment. Despite these potentially significant therapeutic benefits, PegIFN and RBV were poorly tolerated in these "difficultto-treat" and "difficult-to-cure" patients. SAEs, some potentially life threatening, occurred during the course of treatment. Future treatments incorporating direct-acting antivirals that accelerate and enhance virologic response should improve rates of $\mathrm{pTVR}$, but will require strategies to limit toxicity.

Acknowledgment: The authors acknowledge the contributions of the following study coordinators, coinvestigators, and administrative assistants at each of the participating institutions: Andrea Herman, R.N., Carlos Garcia, Michelle Jaramillo, and Rita Lerner at the University of Colorado Denver, Aurora, CO; Janet Mooney at the University of California, Los Angeles, CA; Dulce MacLeod, R.N., at the University of California, San Francisco, LA; Colleen Green and Royanne Dell, R.N., at the University of Virginia, Charlottesville, VA; Patrice Al-Saden at Northwestern University, Chicago, IL; Scott Heese at Columbia University, New York, NY; and Charlotte Hoffman at the Virginia Commonwealth University, Richmond, VA.

\section{Appendix}

The A2ALL Study Group includes Northwestern University, Chicago, IL; University of California - Los Angeles, CA; University of California - San Francisco, CA; University of Colorado Health Sciences Center, Denver, CO; University of North Carolina, Chapel Hill, NC; Epidemiology and Clinical Trials Branch, Division of Digestive Diseases and Nutrition, National Institute of Diabetes and Digestive and Kidney Diseases, National Institutes of Health, Bethesda, MD; University of Michigan, Ann Arbor, MI; Department of Surgery, Columbia Presbyterian Medical Center, New York, NY; University of Pennsylvania, Philadelphia, PA; Department of Internal Medicine, University of Virginia, Charlottesville, VA; and Virginia Commonwealth University, Richmond, VA.

The following individuals were instrumental in the planning, conduct, and/or care of patients enrolled in this study at each of the participating institutions. Columbia University Health Sciences, New York, NY (DK62483): principal investigator, Jean C. Emond, M.D.; coprincipal investigator, Robert S. Brown, Jr., M.D., M.P.H.; study coordinators, Scott Heese, B.A., and Taruna Chawla, M.D. Northwestern University, Chicago, IL (DK62467): principal investigator, Michael M.I. Abecassis, M.D., M.B.A.; coprincipal investigator, Laura M. Kulik, M.D.; study coordinator, Patrice Al-Saden, R.N., C.C.R.C. University of Pennsylvania Health System, Philadelphia, PA (DK62494): principal investigator, Abraham Shaked, M.D., Ph.D.; coprincipal investigator, Kim M. Olthoff, M.D.; study coordinators, Brian Conboy, P.A., M.B.A., and Mary Shaw, R.N., B.B.A. University of Colorado Health Sciences Center, Denver, CO (DK62536): principal investigator, Gregory T. Everson, M.D.; coprincipal investigator, Igal Kam, M.D.; study coordinator, Andrea Herman, R.N. University of California Los Angeles, Los Angeles, CA (DK62496): principal investigator, Johnny C. Hong, M.D.; coprincipal investigator, Ronald W. Busuttil, M.D., Ph.D.; study coordinator, Janet Mooney, R.N., B.S.N. The principal investigator for LADR was Sammy Saab, M.D. University of California San Francisco, San Francisco, CA (DK62444): principal investigator, Chris E. Freise, M.D., F.A.C.S.; coprincipal investigator, Norah A. Terrault, M.D.; study coordinator, Dulce MacLeod, R.N. University of Michigan Medical Center, Ann Arbor, MI (DK62498): principal investigator, Robert M. Merion, M.D.; data coordinating center staff, Anna S.F. Lok, M.D., Akinlolu O. Ojo, M.D., Ph.D., Brenda W. Gillespie, Ph.D., Margaret Hill-Callahan, 
B.S., L.S.W., Terese Howell, B.S., C.C.R.C., Lisa Holloway, B.S., C.C.R.C., Charlotte J. Arrington, M.P.H, Beth Golden, B.S.N, C.C.R.C., Monique Lowe, M.S., and Abby Smith, M.S., University of North Carolina, Chapel Hill, NC (DK62505): principal investigator, Paul H. Hayashi, M.D., M.P.H.; study coordinator, Tracy Russell, M.A. University of Virginia (DK62484): principal investigator, Carl L. Berg, M.D., Duke University; study coordinator, Jaye Davis, R.N., and Colleen Green, P.A. The principal investigator for LADR was Abdullah M.S. Al-Osaimi, M.D. Medical College of Virginia Hospitals, Virginia Commonwealth University, Richmond, VA (DK62531): principal investigator, Robert A. Fisher, M.D., F.A.C.S.; coprincipal investigator, R. Todd Stravitz, M.D.; study coordinators, April Ashworth, R.N., Andrea Lassiter, B.S., and Charlotte Hoffman, R.N. The principal investigator for LADR was Mitchell Shiffman, M.D. Division of Digestive Diseases and Nutrition, National Institute of Diabetes and Digestive and Kidney Diseases, Bethesda, MD: James E. Everhart, M.D., M.P.H., Averell Sherker, M.D., and Jay H. Hoofnagle, M.D.

\section{References}

1. Wiesner RH, Sorrell M, Villamil F; and the International Liver Transplantation Society Expert Panel. Report of the first international liver transplantation society expert panel consensus conference on liver transplantation and hepatitis C. Liver Transpl 2003;9(Suppl):S1-S9.

2. Ghany MG, Strader DB, Thomas DL, Seeff LB. Diagnosis, management and treatment of hepatitis C: an update. Hepatology 2009;49: 1335-1374.

3. Forman LM, Lewis JD, Berlin JA, Feldman HI, Lucey MR. The association between hepatitis $\mathrm{C}$ infection and survival after orthotopic liver transplantation. Gastroenterology 2002;122:889-896.

4. Berenguer M. What determines the natural history of recurrent hepatitis C after liver transplantation? J Hepatol 2005;42:448-456.

5. Charlton M, Wiesner R. Natural history and management of hepatitis C infection after liver transplantation. Semin Liver Dis 2004; 24(Suppl):S79-S88.

6. Fried MW, Shiffman ML, Reddy KR, Smith C, Marinos G, Goncales FL, Jr., et al. Peginterferon alfa-2a plus ribavirin for chronic hepatitis C virus infection. N Engl J Med 2002;347:975-982.
7. Manns MP, McHutchison JG, Gordon SC, Rustgi VK, Shiffman M, Reindollar R, et al. Peginterferon alfa-2b plus ribavirin compared with interferon alfa-2b plus ribavirin for initial treatment of chronic hepatitis C: a randomised trial. Lancet 2001;358:958-965.

8. Hadziyannis SJ, Sette H, Jr., Morgan TR, Balan V, Diago M, Marcellin $\mathrm{P}$, et al. Peginterferon alfa- $2 \mathrm{a}$ and ribavirin combination therapy in chronic hepatitis C: a randomized study of treatment duration and ribavirin dose. Ann Intern Med 2004;140:346-355.

9. Shiffman ML, Di Bisceglie AM, Lindsay KL, Morishima C, Wright EC, Everson GT, et al. Peginterferon alfa- $2 \mathrm{a}$ and ribavirin in patients with chronic hepatitis $\mathrm{C}$ who have failed prior treatment. Gastroenterology 2004;126:1015-1023.

10. Everson GT, Hoefs JC, Seeff LB, Bonkovsky HL, Naishadham D, Shiffman ML, et al. Impact of disease severity on outcome of antiviral therapy for chronic hepatitis C: lessons from the HALT-C trial. HepATOLOGY 2006;44:1675-1684.

11. Poynard T, Colombo M, Bruix J, Schiff E, Terg R, Flamm S, et al. Peginterferon alfa- $2 \mathrm{~b}$ and ribavirin: effective in patients with hepatitis C who failed interferon alfa/ribavirin therapy. Gastroenterology 2009; 136:1618-1628.

12. Everson GT, Trotter J, Forman L, Kugelmas M, Halprin A, Fey B, Ray C. Treatment of advanced hepatitis $\mathrm{C}$ with a low accelerating dosage regimen of antiviral therapy. Hepatology 2005;42:255-262.

13. Crippin JS, McCashland T, Terrault N, Sheiner P, Charlton MR. A pilot study of the tolerability and efficacy of antiviral therapy in hepatitis $\mathrm{C}$ virus-infected patients awaiting liver transplantation. Liver Transpl 2002;8:350-355.

14. Forns X, Garcia-Retortillo M, Serrano T, Feliu A, Suarez F, de la Mata $\mathrm{M}$, et al. Antiviral therapy of patients with decompensated cirrhosis to prevent recurrence of hepatitis $\mathrm{C}$ after liver transplantation. J Hepatol 2003;39:389-396.

15. Thomas RM, Brems JJ, Guzman-Hartman G, Yong S, Cavaliere P, Van Thiel DH. Infection with chronic hepatitis $C$ virus and liver transplantation: a role for interferon therapy before transplantation. Liver Transpl 2003;9:905-915.

16. Carrion JA, Martinez-Bauer E, Crespo G, Ramirez S, Perez-del-Pulgar $S$, Garcia-Valdecasas JC, et al. Antiviral therapy increases the risk of bacterial infections in $\mathrm{HCV}$-infected cirrhotic patients awaiting liver transplantation: a retrospective study. J Hepatol 2009;50:719-728.

17. Gillespie BW, Chen Q, Reichert H, Franzblau A, Hedgeman E, Lepkowski J, et al. Estimating population distributions when some data are below a limit of detection by using a reverse Kaplan-Meier estimator. Epidemiology 2010;21(Suppl 4):S64-S70.

18. Shiffman ML, Ghany MG, Morgan TR, Wright EC, Everson GT, Lindsay KL, et al. Impact of reducing peginterferon alfa-2a and ribavirin dose during retreatment in patients with chronic hepatitis C. Gastroenterology 2007;132:103-112.

19. Sulkowski MS, Shiffman ML, Afdahl NH, Reddy KR, McCone J, Lee WM, et al. Hepatitis $\mathrm{C}$ virus treatment-related anemia is associated with higher sustained virologic response rate. Gastroenterology 2010; 139:1602-1611. 